\title{
On the Extended Clinical Workflows for Personalized Healthcare
}

\author{
Milan Zdravković and Miroslav Trajanović \\ Faculty of Mechanical Engineering, University of Niš, \\ ul. Aleksandra Medvedeva 14, 18000 Niš, Serbia \\ \{milan.zdravkovic, miroslav.trajanovic\}@masfak.ni.ac.rs
}

\begin{abstract}
There are many cases in the clinical practice where using personalized medical products could decrease the cost of treatment and risk of possible complications. However, due to the large costs and long manufacturing lead time, the medical products are customized to the individual patient's needs only in a few critical treatments. One of the main cost factors of the collaboration between the clinical centres and custom medical product suppliers is uptake of human effort in exchange of knowledge between two domains and corresponding issues. In this paper, we use the concepts of the networked enterprises to define the extended clinical workflow which spans the medical and manufacturing practice. We identify the associated systems infrastructure of this workflow and related interoperability issues. The extended workflow is demonstrated on the case study for custom orthopedic implants manufacturing.
\end{abstract}

Keywords: Ontology, Enterprise Interoperability, Supply Chain Management, SCOR.

\section{Introduction}

Current fragmentation of health sciences and medical care along traditional boundaries is considered [1] as artificial and inefficient with respect to many scientific hypotheses that establish the correspondences between the concepts from the different scientific disciplines (biology, physiology, etc.) or clinical specialties (such as cardiology, neurology, etc.). This fragmentation can be considered at modeling level, where reductionist approach (modeling on a dimensional scale, such as organ, tissue, cellular and molecular) dominates over the systemic one [2].

It is foreseen that a more effective approach will integrate the different relevant areas according to the focus of the particular problem, unconstrained by scientific discipline, anatomical subsystem and temporal or dimensional scale [3]. The Virtual Physiological Human (VPH) paradigm [1] is intended to provide a unifying framework that enables and practically benefits from the integration of interdisciplinary data and observations about human's biology. These observations may be collected, organized and shared across the laboratories and hospitals, so that clinical and non-clinical experts can collaboratively interpret, model, validate and understand the data. Thus, this unifying framework is expected to facilitate: 1) integration of 
physiological processes across different length and time scales; 2) integration of descriptive data with predictive models; and 3) integration across disciplines [4]. Then, this integration will eventually lead to the practical benefits of the future healthcare system, such as personalized care solutions; reduced need for experiments on animals; more holistic approach to medicine; and preventative approach to treatment of diseases [4].

The impact of the VPH on industry will first be felt in the medical device and pharmaceutical industries [1]. The prediction sets an interesting assumption that the knowledge relevant to VPH will be integrated faster across the boundaries of all organizations involved in a healthcare (including hospitals, clinical centers, as well as pharmaceutical and manufacturing industries), than within the clinical centers. The prediction is argued by the global distribution of innovation interest and knowledge and developing trend in providing personalized healthcare, which is often related to customization of the medical products.

As an effect of this integration, the traditional clinical workflows will be extended to involve all actors that contribute to delivery of a personalized healthcare, in systematic, efficient way. In addition, the rate of use of custom medical products, such as custom head and neck support systems [5], orthopedic implants [6], patient rooms [7], blood coagulants [8] and others will increase. As a consequence, more and more supply chains, and not only pharmaceutical ones [9] will span the clinical workflows. This effect will facilitate higher degree of customization of the medical products. It reduces the risk, efficiency and cost of treatment, due to increased similarity to the individual patient's anatomy and physiology. For example, standard bone implants are sometimes not sufficient because of abnormal joint anatomy or possible risks of postoperative complications [10], such as aseptic loosening which occurs due to uneven stress distribution on the bone surface. This problem can be addressed by custom design process in which the design of the implant is accommodated to the specific features of the patient's anatomy. However, the traditional approach to supply chain planning cannot be applied in the scenarios of custom medical products manufacturing, due to long delivery times.

Manufacturing of the custom medical products is considered as one-of-a-kind manufacturing, where the customization requirements often affect not only a principal manufacturer but also its suppliers. The manufacturing of a custom medical product could also include high-tech services by different suppliers, which are based on the models which need to be exchanged (for example, the reverse engineering of the missing part of a bone). Typically, some of these services precede supply chain planning phase because their results often determine the basic product's topology.

Because of such a complex scenario, clinicians often choose standard products, even at the cost of sacrificing the above listed benefits of custom ones. Exactly this, not always desirable compromise was the main motivation for the research presented in this paper. The key research problem was identified as "high complexity of the supply chain planning and execution in custom medical products manufacturing".

In our research, this problem is addressed by combining practices of collaborative networked organizations with clinical practices. As one of the results, an extended clinical workflow is proposed. Besides the traditional activities of the clinical 
practice, this extended workflow also encompasses planning, decision making, design, sourcing and manufacturing of custom medical products. It also considers systems' and knowledge infrastructures which facilitate the efficient execution of this extended workflow. In a way, the models and knowledge required to resolve interoperability issues of such a workflow can be considered as extension to VPH paradigm, because the topology and design of a custom medical product correspond to physiological and anatomical features of a patient, represented by VPH models.

The remainder of this paper is structured as follows. In part 2, the traditional clinical workflow is described in context of Electronic Health Record, a paradigm which is often used to integrate patient specific information throughout the history of medical care delivery. Part 3 presents the extended clinical workflow, associated resources, namely systems infrastructure; and analysis of interoperability issues of such infrastructure. In part 4, a study is presented, on the case of manufacturing the custom orthopedic implant for diagnosis of bone cancer of tibia. Finally, in part 5, the main conclusions are drawn.

\section{Electronic Health Records and Clinical Workflows}

In practice, the clinical workflows are often defined in context of Electronic Health Record (EHR). Health Information Management Systems Society's (HIMSS) defines EHR as "the longitudinal electronic record of patient health information generated by one or more encounters in any care delivery setting..."

Many benefits from maintaining EHR are expected, such as automation and streamlining of the clinical workflow, evidence-based decision support for diagnosis or treatment prescription (based on accurate and complete record of a clinical patient encounter), support to other care-related activities such as billing, reporting and quality management. An EHR enables the hospital administrator to extract the billing data, the physician to assess the effectiveness of treatments, a nurse to monitor treatment and reactions and a researcher to analyze the efficiency of medications.

One of the main issues of EHR is the fact that it is not a record of all care provided to the patient in all facilities over time. It is generated and maintained within the single medical centre. Even so, one of the greatest challenges of maintaining EHR arises from the collaborative effort in collection and analysis of its data. Namely, medical centers can be considered as complex enterprises. They typically consist of multiple healthcare facilities, such as affiliated hospitals and clinics, diagnostic and treatment centers and laboratories. Managing all of these departments implies the complex business processes, for which EHR is fully associated.

\subsection{Clinical Workflow}

In a way, EHR is the patient specific representation of a clinical workflow, combined with information (from the observations) collected in the course of this workflow. It

${ }^{1}$ http://www.himss.org/ASP/topics_ehr.asp 
typically connects administrative data with information from the relevant health information systems. Figure 1 illustrates a simplified representation of a clinical workflow for inpatient care.

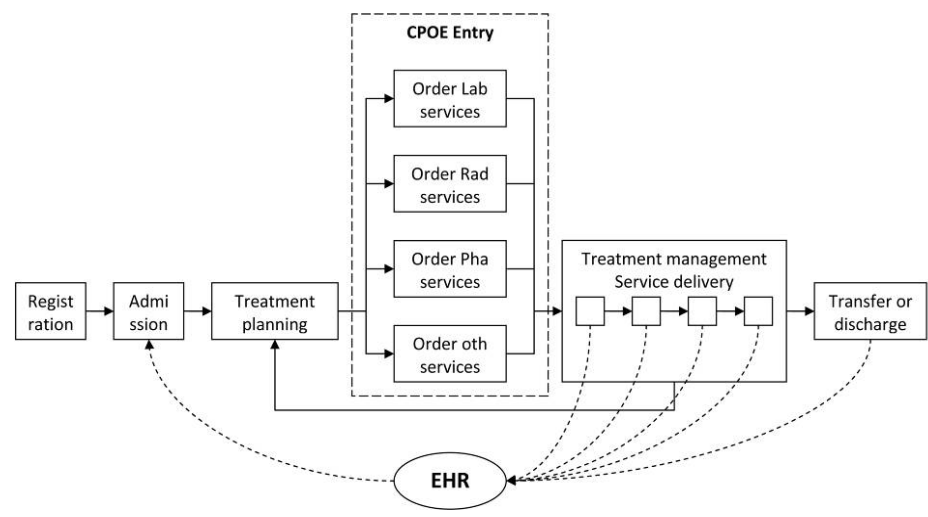

Fig. 1. Simplified representation of the clinical workflow for inpatient care

Registration, admissions, discharge, and transfer (RADT) data are the key components of EHRs. These data include vital information for accurate patient identification and assessment, such as name, demographics, employer information, etc. The registration portion of an EHR contains a patient identifier (master patient index MPI), which is identifiable only inside the organization in which the EHR is maintained. EHR record for a specific patient is recovered during his/her admission. Admission notes are added in case that inpatient care need to be provided and include patient's status, reasons why the patient is being admitted and initial instructions for patient care.

An EHR can be considered as patient specific RADT data, integrated with respective information from Laboratory Information Systems, Radiology Information Systems, Electronic clinical documentation systems and pharmacy systems. This integration is carried out by Computerized Physician Order Entries (CPOE) which permits clinical providers to electronically order laboratory, pharmacy, radiology and other services.

CPOE entries are initially entered according to first patient observations and treatment plan. Once the treatments are launched, namely during and after entered orders execution, it may become necessary to require additional actions, such as preoperation planning, other tests, etc. When all treatments (in one or multiple iterations) are carried out, the patient is discharged or transferred. All treatments' results and notes, including the administrative data on the discharge and transfer are added to a patient's EHR.

\section{Extended Clinical Workflows and Associated Systems Infrastructure}

In general, two of the most critical non-technical barriers to customization are: 1) lack of efficiency of manufacturing enterprise to handle one-of-a-kind production tasks; 
and 2) lack of efficiency in transfer of multi-disciplinary knowledge, required for the design of custom product. Manufacturing enterprises refine their designs for simplicity and cost; they design their workflows for volume manufacturing. Hence, by default, they are not capable to handle one-of-a-kind manufacturing tasks efficiently.

One-of-a-kind manufacturing is considered as a case for the Virtual Enterprises. Virtual Enterprise (VE) is a temporary network of independent organizations, who join together quickly to exploit fast-changing opportunities and then dissolve [11]. It is characterized by a short-living appearance of a supply chain, capable to produce low volume of high variety of products, by drawing from the loosely-coupled, heterogeneous environment of available competences, capabilities and resources. This environment is sometimes called Virtual Breeding Environment (VBE), defined as a pool of organizations and related supporting institutions that have both the potential and the will to cooperate with each other, through the establishment of a long-term cooperation agreement and interoperable infrastructure [12]. In our research, VBE and VE paradigms are used to propose the interoperable infrastructure which will support the extended clinical workflows for custom medical products.

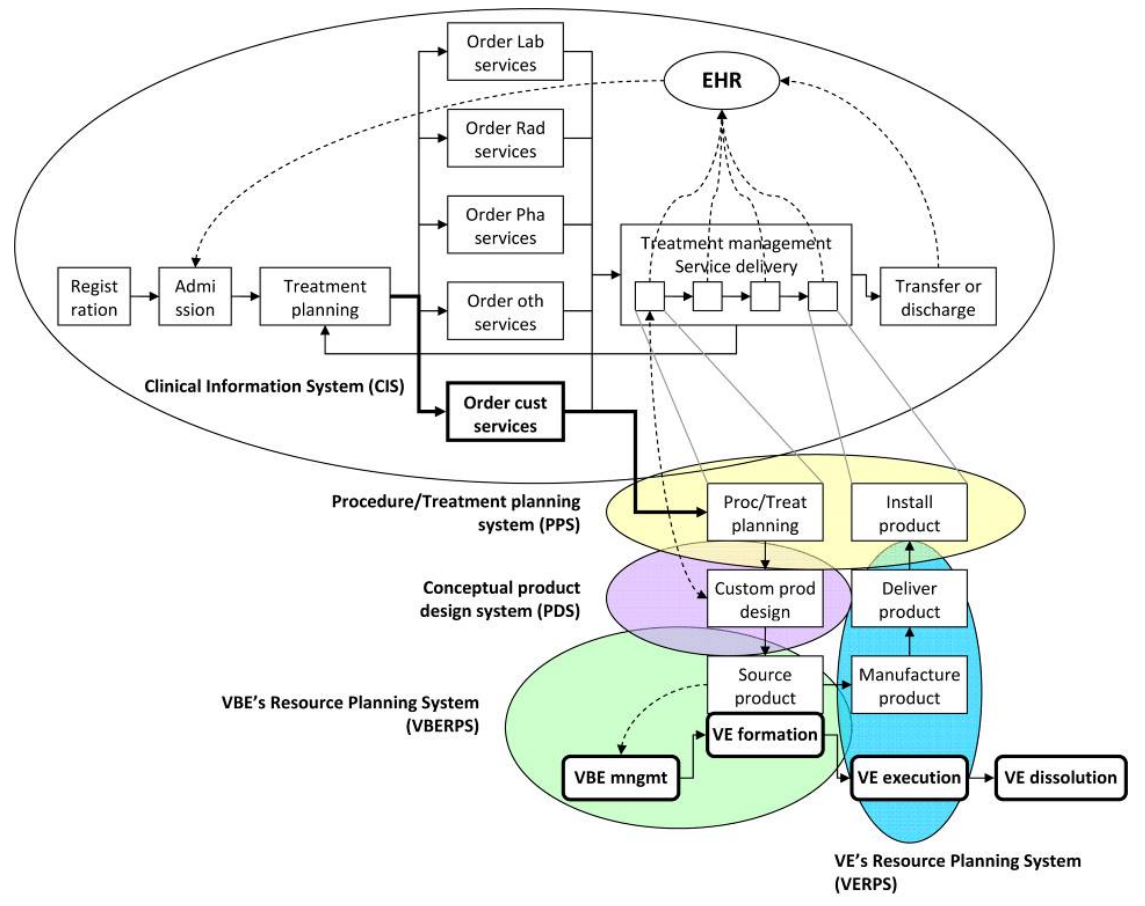

Fig. 2. Simplified representation of the extended clinical workflow

In traditional settings, the workflow for manufacturing of custom medical products includes many human analysis and decisions, such as interpretation and analysis of CT scans and lab results, mechanical analysis, collecting inputs and approvals, etc. The lack of efficiency to adapt their traditional workflows to these activities becomes 
even more critical when enterprises are required to subcontract the different parts or services suppliers. All this human involvement includes a number of interactions between different experts in which functional (medical), organizational and other perspectives to the custom manufacturing need to be considered. Hence, efficient design elaboration and mutual understanding on the complex variety of issues require involvement of experts with multi-disciplinary skills and knowledge.

In order to overcome the barriers above, the extended clinical workflow and associated systems infrastructure is proposed. Figure 2 illustrates simplified representation of the extended clinical workflow with associated systems, foreseen as facilitators of this workflow.

Traditional clinical workflows (see Fig. 1) are based on the order-delivery service sequences and/or cycles, where these services are related to specialized observations and/or treatments. In the extended clinical workflow, the manufacturing of a custom medical product (with all associated services) is considered as a single service which can be ordered by using CPOE entry in the Clinical Information System. For fulfillment of this entry, six key activities are required: 1) procedure/treatment planning; 2) custom product design; 3) source product; 4) manufacture product; 5) product delivery; and 6) product installment. While procedure/treatment planning and installment are fully integrated in the traditional clinical workflow, other activities are carried out in the shared or environment of VBE, which is the main supplier of the clinical center for custom medical products of a certain type. Each of the activities of the clinical workflow should be facilitated by specific (hypothetical) system, as it is illustrated appropriately on Figure 2.

\subsection{On the Systems Infrastructure for Extended Clinical Workflows}

The design of the custom medical product is never considered in isolation from the procedure of its installment or a treatment method; as it must take into account the constraints and requirements of the specific intervention (e.g. surgery). Typically, the procedure/treatment planning is not facilitated by the information system or a tool. The decisions made in this phase are used to select from a range of standard medical products. In most of the cases, the problem of selecting a standard product is trivial.

However, in case of custom medical products, the Procedure/Treatment Planning System (PPS) is considered as essential, because its output is later used by the system for a product design, to define the main features (mechanical, geometrical, chemical, etc.) and topology of the custom product. Namely, in great most of the cases, there are strong correspondences between these features and steps, micro-steps and assets used in the installment of the custom medical product or treatment process. Hence, PPS is intended to be used for developing and generating a kind of a process model, which significant features will be then mapped to the features of the custom medical product conceptual model. The product model is considered as conceptual because it includes only features which are necessary and sufficient for establishment of the abovementioned correspondences (both with PPS and VBERPS) and is designed by using the Conceptual Product Design System (PDS). 
VBE Resource Planning System (VBERPS) is foreseen to be used in the sourcing step of the extended clinical workflow, where the Virtual Enterprise (VE) is formed from the VBE, according to the features of the conceptual product model. VBERPS is expected to have access to the relevant information for determining the capacity and availability of each enterprise of VBE to carry out a specific role, according to the conceptual product model (including its Bill of Material) and associated requirements, defined by the features of the respective parts.

Finally, the lifecycle of this VE is managed by using VE Resource Planning System (VERPS), which is typical an ERP system of the VE's focal partner.

\subsection{Systems Interoperability in Extended Clinical Workflows}

Interoperability is one of the main enablers of the extended clinical workflow, because it facilitates the flexible collaboration; it reduces the time needed for the setup and discontinuation of the VE. Given the high requirements for the workflow's efficiency, it is of outmost importance to remove as many as possible preconditions for the collaboration and requirements for any kind of previous agreements in exchange of relevant information between its actors. Exactly these preconditions and requirements are considered as some of the most difficult challenges in implementing the extended clinical workflow.

Systems interoperability issues of the extended clinical workflow can be easily identified in the intersections of the systems' scopes from the proposed infrastructure (see Fig.2). They are related to the interoperations and data exchanges illustrated on Figure 3.

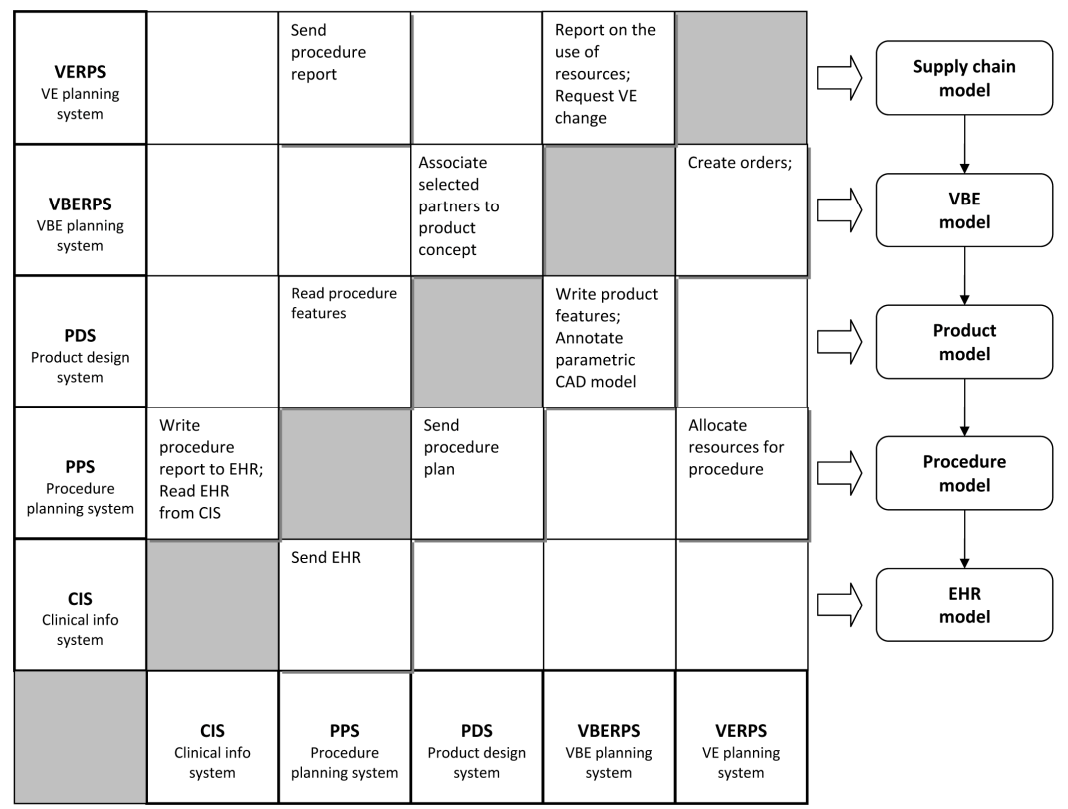

Fig. 3. Systems interoperations and models infrastructure in the extended clinical workflow 
Since the capacity to interoperate is unidirectional capability of systems, the twofold consideration of each interoperation is assumed. Namely, in every interoperation between two systems, each of these two systems must exhibit the non-interrelated, independent capabilities to send and receive (and interpret) the exchanged messages or invocation requests. However, the minimum requirement is considered as use of pre-determined or pre-selected dictionaries, vocabularies or even formal models (e.g. ontologies) in formulating these messages and requests, so they can be correctly interpreted. Thus, a Model-Driven Architecture for resolution of the interoperability requirements is foreseen. The conceptual view of involved models and dependencies between these models is illustrated on Figure 3.

The most difficult interoperability challenge of the extended clinical workflow is related to establishment of the correspondences between two, quite different domains of manufacturing and clinical practice. While the manufacturing domain knowledge is embedded in VBE and supply chain models and partially, in product model, the clinical practice is formalized by the EHR and procedure models.

Today's EHR records often suffer from the vendor-specific realizations of patient record data sets which rarely accommodate to the controlled terminologies [13]. However, the inefficiency of the clinical workflows which extend beyond the boundaries of a single medical centre is establishing EHR interoperability as one of the main requirements for health information systems. The issues of EHR interoperability are addressed by combining the standards for clinical vocabularies and healthcare message formats; with EHR ontologies (i.e., content and structure of the data entities, both from vocabularies and messages, in relation to each other).

Procedure model can be considered as a process model, as it is intended to formalize a set of actions in a linear or more complex flows, that could also include the equivalents of the error handler and compensation blocks from the workflow management.

\section{Case Study - Extended Workflows for Custom Orthopedic Implants Manufacturing}

The research of custom orthopedic implants manufacturing is typically focused to direct fabrication technologies [14]. Namely, direct manufacturing of high-strength materials provide far greater efficiency in one-of-a-kind runs for producing a finished custom implant than the conventional manufacturing technologies.

Depending on nature of the bone trauma, the custom orthopedic implant can be assembled of some of different types and designs of fixtures and scaffolds. In addition, some services may be associated to the product manufacturing and/or implementation, such as: pre-operation planning, reposition simulation, digital reconstruction, remodeling, analysis of biomechanical properties of the implant, sterilization, ethical review, product certification and others. For example, in case of bone cancer of tibia (larger of the two bones in the leg, below the knee), the missing part of the bone is replaced with the scaffold, which is enforced with the inner fixture. The scaffold is designed on the basis of bone geometry, which is digitally 
reconstructed from CT scans. Geometry and topology of inner fixture is designed on the basis of diagnosis and pre-operation plan, developed by surgeon. The process of manufacturing of the custom part is associated also with review of the design by the clinics ethical committee and analysis of biomechanical properties. Obviously, in above scenario, efficiency brought by the use of additive manufacturing is only a tip of the iceberg. It needs to be complemented by the effectiveness of the appropriate collaboration infrastructure which will facilitate all planning, sourcing, manufacturing and delivery aspects.

In our case, we propose to extend the clinical workflow for treatment of tibia bone cancer with the manufacturing of the custom implant parts and provision of the associated services. This is carried out within the VBE, which consists of the enterprises, capable, certified and competent to deliver a manufactured product and/or to provide associated services. VBE is organized as a cluster and technically coordinated by the brokering enterprise (broker). Each case of supply of the product and associated services is considered as a case of VE. In this case, the systems and models infrastructure, proposed in Section 3 is instantiated, as it is illustrated on Figure 4.

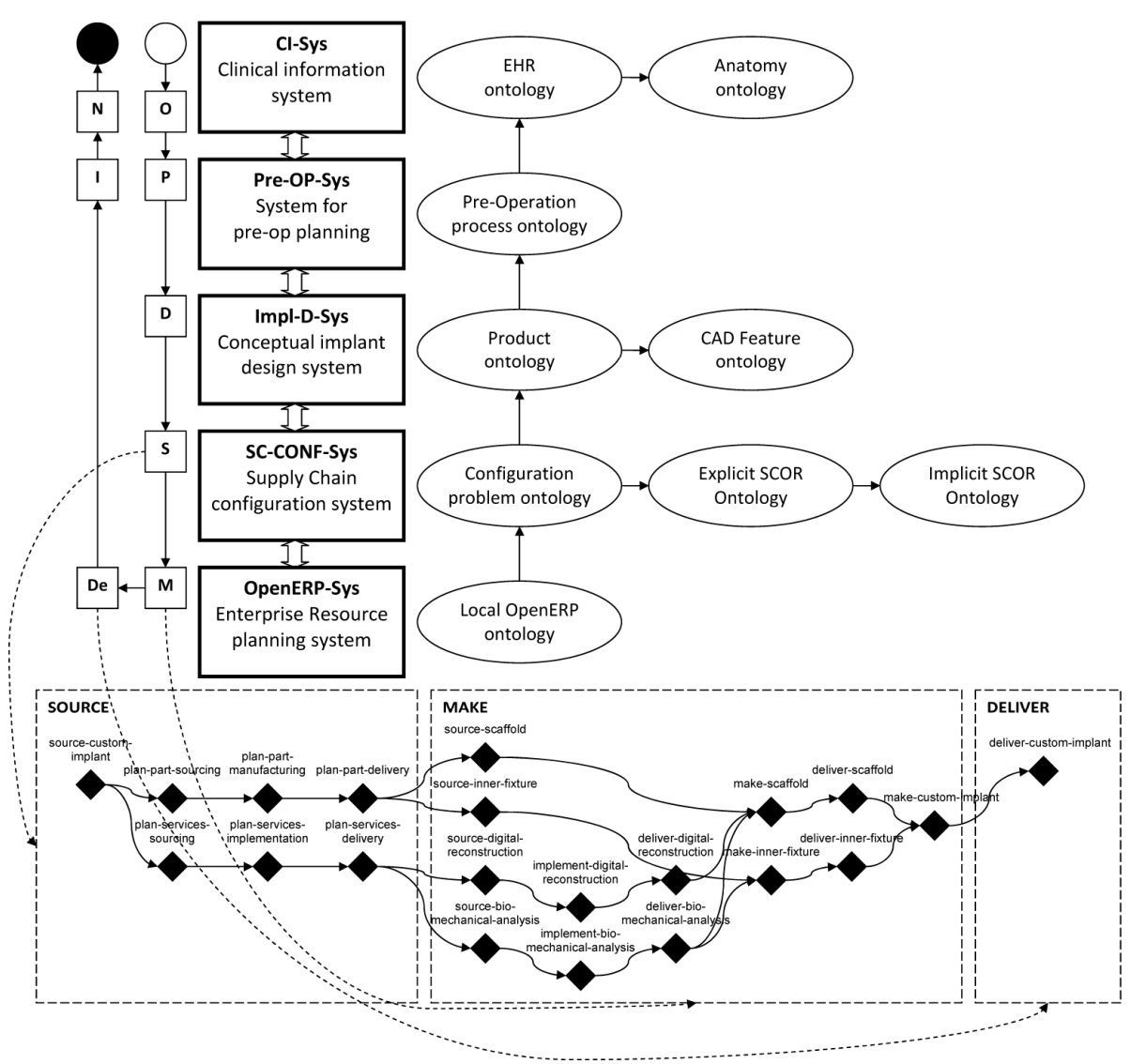

Fig. 4. Systems and models infrastructure for custom orthopedic implants manufacturing 
The proposed infrastructure is implemented by using semantic applications and information systems which exploit the framework of inter related ontologies (models), that consist of the different domain (system) concepts and logical relationships between those [6]. The ontological framework corresponds to the assumed models infrastructure for extended clinical workflows and it is managed by the instances of the relevant assumed systems.

Clinical information system (CI-Sys) is used to create the order (O) for the custom implant manufacturing and to trigger the execution of the next $(\mathrm{N})$ order of the extended clinical workflow, upon the installation of the implant.

System for pre-operation planning (Pre-OP-Sys) is used to plan (P) this installation (I). Pre-operation planning is based on the location and the arrangement of anatomical structure parts within the human body, expressed in quantitative or qualitative way (by using spatial orderings such as superior, anterior, lateral, etc). This arrangement can be formalized by appropriate anatomical ontology [15]. When operation is planned, the relevant spatial features are used to determine the features of the microsteps which are carried out during the surgery, such as bone screw entry angles, fixture-bone assembly contact locations, etc. Hence, relevant properties of the spatial relations can be exploited for automated reasoning [16], which assists pre-operation planning process. In order to make this possible, Pre-OP-Sys need be capable to infer the spatial relations and corresponding micro-steps features, by exploiting previously established logical correspondences between anatomical ontology and pre-operation process ontology (model).

Above-mentioned spatial relations are also relevant for the custom implant design (D), which is facilitated by Impl-D-Sys system. These relations provide formal definitions of the geometry restrictions which are typically considered when design of the orthopedic implant is determined. For example, the angle between distal and proximal part of the inner fixture depends on the specific arrangement of bones and joints. Impl-D-Sys is a semantic application which formalizes parthood relationships of the product (Bill Of Material - BOM) and features of the respective parts and subassemblies. BOM also include relevant services.

Based on the product's topology and manufacturing or delivery strategies of each product part (including the services), a sourcing (S) strategy, namely the supply chain configuration is generated by SC-CONF-Sys application. SC-CONF-Sys is based on SCOR reference model for supply chain operations [17], a standard approach for analysis, design and implementation of core processes in supply chains. SC-CONFSys is semantic application which uses SCOR ontologies at two levels of conceptualization. While implicit SCOR ontology is used to enable interoperation of the SC-CONF-Sys with proprietary SCOR tools, explicit SCOR ontology is expressive domain ontology which defines the meanings of the implicit SCOR entities and thus, it facilitates interoperation of SC-CONF-Sys with other enterprise applications [18]. The supply chain configuration is based on the common rules related to the orderings of SCOR source, make and delivery processes in the different cases of the manufacturing strategies: make-to-stock, make-to-order and engineer-toorder; and a capacity of the supplier to deliver the desired part. At this moment, the capacity is evaluated only by checking the part production schedules of the suppliers through the semantic queries to the local ontologies of their information systems. 
Exactly this last feature of the SC-CONF-Sys application demonstrates how the planning processes of custom orthopedic implants manufacturing could benefit from the semantic interoperability of the systems. Namely, during the process configuration, all local ontologies (representing their Enterprise Information Systems) of all registered manufacturing enterprises of the VBE, are queried by the SC-CONFSys for the production schedules of a given part. Then, based on the part availability at the calculated time, the selected enterprises are automatically assigned to specific process categories. Besides selection process, which is carried out on the basis of above criteria, corresponding semantic relationships between the SCOR ontologies and local ontologies of the EISs of the VBE partners can also facilitate planning of sourcing, manufacturing and delivery of custom product parts at all levels of BOM, as early as in the supply chain process configuration phase.

\section{Conclusions}

Extended clinical workflow aims at complementing the clinical practice with functions which are typically considered as external to the conventional clinical workflows. These functions extend the scope of the clinical practice and they are: procedure/treatment planning (in context of custom product implementation), conceptual custom product design, sourcing and implementation. In broader sense, even manufacturing and delivery can be considered in this extended scope. The main objective is to facilitate efficient application of custom medical products in daily practice. The interoperability challenges implied by the need to resolve many crossdomain issues are addressed by the high-level system and models infrastructure. This infrastructure is expected to enable execution of the processes that span the boundaries of the clinical centre and enterprises from the VBE.

The above assumptions are, to a certain extent validated in a case of manufacturing of custom orthopedic implants. Presented case confirms the hypothetical systems and models infrastructure and instantiates it by realizing the assumed functionality and purpose.

It is expected that the proposed infrastructure could reduce the lifecycle of the VE for custom orthopedic implant manufacturing to 4-8 days. This is considered as acceptable period for many cases of trauma, especially when having in mind that delivery lead time for custom orthopedic implants, even when manufactured by using additive technologies can reach up to 3 months [19]. The estimation of the saved time is based on the fact that integrated infrastructure practically automates the process configuration phase of VE lifecycle and exchange of information between relevant systems, by removing the need for complex technical preconditions so this exchange can occur and by minimizing the human effort in relevant knowledge and information exchanges. Thus, it significantly reduces the time typically needed for supply chain planning.

\section{References}

1. Fenner, J.W., Brook, B., Clapworthy, G., Coveney, P.V., Feipel, V., Gregersen, H., Hose, D.R., Kohl, P., Lawford, P., McCormack, K.M., Pinney, D., Thomas, S.R., Van Sint Jan, S., Waters, S., Viceconti, M.: The EuroPhysiome, STEP and a roadmap for the virtual physiological human. Philosophical Transactions of The Royal Society A Mathematical Physical and Engineering Sciences (July 2008) 
2. Clapworthy, G., Viceconti, M., Coveney, P.V., Kohl, P.: The virtual physiological human: building a framework for computational biomedicine I. Editorial. Philos. Transact. A Math. Phys. Eng. Sci. 366(1878), 2975-2978 (2008)

3. Welsh, E., Jirotka, M., Gavaghan, D.: Post-genomic science: cross-disciplinary and largescale collaborative research and its organizational and technological challenges for the scientific research process. Phil. Trans. R. Soc. A. 364, 1533-1549 (2006)

4. VPH roadmap. Seeding the EuroPhysiome: a roadmap to the virtual physiological human (2008), http: / / www . europhysiome.org

5. Bentel, G.C., Marks, L.B., Sherouse, G.W., Spencer, D.P.: A customized head and neck support system. International Journal of Radiation Oncology, Biology and Physics 32(1), 245-248 (1995)

6. Zdravković. M., Trajanović, M., Stojković, M., Vitković, N., Mišić, D.: A case of using the Semantic Interoperability Framework for custom orthopedic implants manufacturing. Annual Reviews in Control 36(2) (2012)

7. Yassine, A., Kim, K.C., Roemer, T., Holweg, M.: Investigating the role of IT in customized product design. Production Planning \& Control 15(4) (2004)

8. Hess, J.R., Holcomb, J.B., Hoyt, D.B.: Damage control resuscitation: the need for specific blood products to treat the coagulopathy of trauma. Transfusion 46(5), 685-686 (2006)

9. Puschmann, T.: Customer relationship management in the pharmaceutical industry. In: 34th Annual Hawaii International Conference on System Sciences, January 3-6 (2001)

10. Keenan, J., Chakrabarty, G., Newman, J.H.: Treatment of supracondylar femoral fracture above total knee replacement by custom made hinged prosthesis. Knee 7, 165-170 (2000)

11. Browne, J., Zhang, J.: Extended and virtual enterprises - similarities and differences. International Journal of Agile Management Systems 1(1), 30-36 (1999)

12. Sánchez, N.G., Apolinar, D., Zubiaga, G., Atahualpa, J., González, I., Molina, A: Virtual Breeding Environment: A First Approach to Understanding Working and Sharing Principles. In: Proceedings of the 1st International Conference on Interoperability of Enterprise Software and Applications, Geneva, Switzerland, February 23-25 (2005)

13. Harris, M.R., Ruggieri, A.P., Chute, C.G.: From Clinical Records to Regulatory Reporting: Formal Terminologies as Foundation. Health Care Financing Review 24(3), 118 (2003)

14. Gibson, I., Harrysson, O.L.A.: Direct Fabrication of Custom Orthopedic Implants Using Electron Beam Melting Technology. In: Advanced Manufacturing Technology for Medical Applications: Reverse Engineering, Software Conversion and Rapid Prototyping (2006)

15. Burger, A., Davidson, D., Baldock, R.: Anatomy Ontologies for Bioinformatics. Principles and Practice, 1st edn. Computational Biology, vol. 6, XVI, 356 p. (2008)

16. Schulz, S., Hahn, U.: Parts, Locations, and Holes - Formal Reasoning about Anatomical Structures. In: Quaglini, S., Barahona, P., Andreassen, S. (eds.) AIME 2001. LNCS (LNAI), vol. 2101, pp. 293-303. Springer, Heidelberg (2001)

17. Stewart, G.: Supply-chain operations reference model (SCOR): the first cross-industry framework for integrated supply-chain management. Logistics Information Management 10(2), 62-67 (1997)

18. Zdravković, M., Panetto, H., Trajanović, M., Aubrey, A.: An approach for formalising the supply chain operations. Enterprise Information Systems 5(4), 401-421 (2011)

19. Christensen, A., Chen, D.: Personalizing Orthopedic Implants, MDDI Online (2008), http: / /www.mddionline.com/article/ personalizing-orthopedic-implants 